\title{
THE PATHOLOGY OF LYMPH NODE ENLARGEMENT
}

\author{
By L. Woodhouse Price, M.A., M.D.(Cantab.) \\ Director of the Pathological Department, The Royal Cancer Hospital, London
}

Many different aetiological factors contribute to the enlargement of lymph nodes; in some cases the cause is obvious, in others obscure. The enlargement may be focal, regional or generalized, or the first two types may progress to the third. The enlargement may be the only clinical manifestation of disease or it may be accompanied by various signs and symptoms, such as pain, pyrexia, exanthemata, changes in the blood picture or pressure effects on viscera.

The histological differential diagnosis of lymph node enlargement depends upon a proper appreciation of the normal structure of a lymph node and of the various changes which are common to several or peculiar to certain specific types of pathological processes. In some cases a consideration of the clinical condition alone is sufficient to establish a correct diagnosis. This applies especially to lymph node enlargement associated with inflammatory lesions, acute specific fevers and chronic infective granulomata. In other cases the. aid of histology and haematology is essential. The latter group comprises the primary lymphadenopathies and metastatic tumour deposits.

An investigation as to the cause of lymph node enlargement demands an account of the clinical appearance of the patient, the determination of the size, consistency and distribution of the enlarged nodes and a detailed report on the histology of a node excised for biopsy purposes, to which it is often advisable to add the macroscopic appearance of the hemisected node. In certain diseases the concomitant pathological changes in other organs and tissues have to be considered also.
Histology of the Normal Lymph Node. The microscopic appearance is by no means uniform but a generalized or fundamentally characteristic histological picture can be deduced from a study of a large number of lymph nodes excised for biopsy purposes and preserved in various fixative solutions from which paraffin sections are prepared and stained by several different technical methods.

It so happens that certain component parts of a lymph node are accentuated under the influence of certain morbid processes, particularly inflammatory conditions and the reticuloses. Hence, paradoxically, the normal structure is more clearly appreciated from a consideration of the histological appearances of lymph nodes which are slightly abnormal.

The lymph node is a spheroidal, ovoid or reniform body surrounded by a delicate fibrous capsule and composed of certain characteristic components which are clearly recognizable and which can be resolved into three essential constituents, namely, the lymphadenoid parenchyma, the lymphadenoid follicles and the lymphatic sinusoids. The majority of lymph nodes are supported by a surrounding matrix of adipose tissue, but certain homologous lymphoid structures are distributed in the subepithelial region of the buccal, nasal and pharyngeal mucosae and the submucous tissues of the alimentary canal.

The Capsule. Fully developed lymph nodes are completely surrounded by a delicate fibrous capsule which is perforated by afferent and efferent blood vessels and by afferent and efferent lymphatic channels. In nodes which are only partially developed the capsule is incomplete. Such nodes on hemisection in the 
longitudinal axis present a C-shaped or horseshoe outline, the convexity being covered by a capsule while the concavity is filled with adipose tissue into which lymphocytes appear to be diffusely permeating. This peculiar structure has an important bearing on the spread of metastatic tumours.

The Lymphadenoid Parenchyma. This consists essentially of a matrix of lymphocytes and lymphoblasts. Under conditions of reticulo endothelial hyperplasia isolated elements or small groups of reticulo endothelial cells are found lying free in the matrix. This component of the fundamental composition of the normal lymph node becomes encroached upon by reticuloses of either the follicles or the sinusoids. It is particularly affected in certain lipodystrophies and shares in the general disorganization of normal lymphadenoid architecture in Hodgkin's disease. On the other hand, the lymphadenoid parenchyma predominates over both follicles and sinusoids in lymphatic leukaemia and in the lymphosarcomata.

The Lymphadenoid Follicles. Around the periphery of the node there are circumscribed spheroidal or ovoid bodies known as the lymphadenoid follicles, the lymph follicles or the germ centres of Flemming. These consist of a core of reticulo endothelial cells surrounded by several concentrically laminated layers of lymphocytes. Normally, the central zone of reticulo endothelial cells is inconspicuous but a hyperplastic reaction is readily invoked by any slight inflammatory process. Nodes excised for biopsy purposes are, ipso facto, to some extent pathological. However inconspicuous they may be, the presence of the central zone of reticulo endothelial cells and the lamination of the surrounding lymphocytes are essential to the composition of the true follicle.

It is readily understandable that the plane of the section may pass through the boundary zone of a complete follicle so that only a circumscribed aggregate of lymphocytes appears in the microscopic field; such collections are referred to as ' pseudofollicles.'

The Lymphadenoid Sinusoids. The lymphadenoid parenchyma is permeated by a meshwork of lymphatic channels which intercommunicate and which constitute the sinu- soids. 'These are divisible anatomically into the subcapsular group which forms a lymphatic lake between the capsule and the lymphadenoid parenchyma, and the medullary group in the centre of the node. Between these two groups there is free intercommunication of lymphatic channels. The subcapsular sinus is joined by several afferent lymphatic channels which perforate the capsule around the periphery of the ovoid and reniform nodes and around the convexity of the C-shaped and horseshoe nodes. At the hilum of the node an efferent lymphatic channel emerges, and at this point the nutrient vessels find access and egress. Valves are discernible in the extra capsular zone in both afferent and efferent lymphatic channels.

The lymphadenoid sinusoids are lined by reticulo endothelial cells which also traverse their lumina, converting them into a spongelike meshwork whose interstices contain circulating lymph.

Under normal conditions this sinusoidal meshwork is difficult to detect in routine histological preparations but it is readily discernible under conditions of reticulo endothelial hyperplasia and catarrhal conditions of the sinusoids. In certain lipodystrophies the sinusoids become distended with lipoid containing cells, and in lymphogenous metastasis of malignant tumours they contain plugs of specific neoplastic cells.

Pathological Changes in Lymph Nodes. Various types of cellular response are encountered in lymph nodes as component manifestations of various diseases. Any or all of the three essential elements of the lymph node may be affected to a greater or less extent. Thus the follicles and sinusoids may become unduly prominent due to reticulo endothelial hyperplasia or they may become obliterated or modified by cellular proliferation of the lymphadenoid parenchyma. Pathological changes may also be caused by the advent of extraneous cells not normally found in the lymph node, as exemplified by the malignant epithelial cell in metastatic carcinoma.

The pathology of lymph node enlargement may conveniently be described under the following main headings :- 
I. Acute Lymphadenitis.

2. Chronic Lymphadenitis.

3. The Reticuloses and Lipodystrophies.

4. Primary Lymphadenopathies.

5. Secondary Lymphadenopathies.

6. The differential diagnosis of specific cases of lymph node enlargement.

\section{Acute Lymphadenitis}

Aetiological factors are exemplified by acute infection of the skin with pyogenic cocci, of the tonsils with streptococci, of the nasopharynx with the Klebs-Loeffler bacillus and of the urethra with Neisserian organisms. Macroscopically the regional lymph nodes present the classical signs of inflammation, accompanied in fulminating cases by acute lymphangitis. On inspection the affected nodes are enlarged, soft in consistency, hyperaemic and greyish-pink in colour. On hemisection they appear semi-diffluent. Yellowish areas of necrosis are present in virulent infections such as diphtheria and typhoid fever

Histologically, all the stages of acute inflammation, terminating in resolution, similar to those seen in the pneumonic lung, may be encountered. In the milder types of infection there are seen proliferating, lymphocytes with marked reticulo endothelial hyperplasia of both lymphadenoid follicles and sinuses. It is important that the presence of mitotic figures in such hyperplastic nodes is not mistaken for an indication of malignancy. The distinction is based on the absence of nuclear hyperchromia and reduplication and on the absence of heterotype mitoses in the inflamed node.

The capillaries are hyperaemic, and, in nonsuppurating examples, there is a diffuse interstitial granulocytic infiltration of the lymphadenoid parenchyma resulting from leucocytic diapedesis.

In the fulminating types of infection suppuration occurs with the formation of multiple miliary or large solitary abscesses. In this event the supervention of periadenitis leads to a matting together of individual lymph nodes. Acute inflammation of the surrounding tissues follows and is succeeded by necrosis and sloughing with perforation of the skin and the formation of sinuses.
In cases which do not proceed to suppuration, complete or partial resolution follows. Resolution is preceded by deposition of fibrin in the sinuses, followed by fibrosis and subsequent phagocytosis, and the affected node may eventually be restored to its normal morphology. In some cases, however, fibrosis persists owing to the continued presence of infecting organisms of impaired virulence, and the acute form of lymphadenitis leads to its chronic counterpart.

\section{Chronic Lymphadenitis}

Certain non-specific forms of chronic lymphadenitis are more conveniently considered in relation to reactionary reticuloses. Those forms which are due directly to specific bacterial infections may be grouped as follows :-

(a) Chronic Lymphadenitis following Acute Lymphadenitis. In this lymphadenoid manifestation of infection there is incomplete resolution of the acute form, with persistence of the infecting micro-organisms within the lymphadenoid parenchyma. Such organisms appear to have assumed diminished virulence, and indeed they are to some extent walled-off from surrounding tissues by the capsular fibrosis which plays so prominent a part in the histological picture.

Histologically the appearances are similar to those seen in reactionary reticuloses and comprise essentially a hyperplasia of the reticulo endothelial elements of the lymph nodes. In addition, there is often some degree of interstitial fibrosis, and, as above stated, capsular fibrosis. The accompanying interstitial infiltration with plasma cells, eosinophilous cells and histiocytes may lead to difficulty in determining the diagnosis. Lymph nodes affected by chronic bacterial lymphadenitis have erroneously been diagnosed as gummata. The histological diagnosis rests on the demonstration of pyogenic cocci in sections specifically stained for micro-organisms and on the absence of gummatous areas of vascularized necrotic tissue, the absence of specific giant cells and the absence of endarteritis obliterans.

\section{(b) Tuberculous Lymphadenitis. Infection}


occurs primarily in the teeth, tonsils, adenoids, lungs or intestinal tract, and the specific type of lymphadenitis results either from lymphogenous or haematogenous dissemination. In lymph stream dissemination the lymph node lesions are peripheral and only regional nodes are involved. In blood stream dissemination the lymph node lesions are central and several widespread groups of nodes are involved.

Macroscopically, the affected nodes are enlarged, firm in consistency and greyish in colour. On hemisection the cut surface shows translucent grey points where miliary tubercles have developed. At a later stage when caseation has occurred the cut surface appears opaque and yellow. If suppuration supervenes as a result of superadded infection with secondary pyogenic cocci, there is a fluid purulent exudate which flows from the incised node.

Microscopically, there is at first a general reticulosis affecting both lymphadenoid follicles and sinuses. Localized groups of endothelial cells appear, surrounded by proliferating lymphocytes. These constitute the miliary tubercle. An avascular necrosis soon occurs and miliary tubercles become confluent. Circumscribed areas of caseation become apparent, at the margins of which the characteristic ' giant cell' with peripheral nuclei is discernible in greater or smaller numbers. These cells usually show a C-shaped ring of large, clear, slightly reticulated nuclei occupying approximately threequarters of the circumference of the cell cytoplasm. The peripheral margin of the cell is somewhat of stellate form. The lymphadenoid matrix contains lymphocytes, plasma cells and fibroblasts.

When the lesions in the affected lymph nodes become more advanced, the histological picture is modified by the development of massive caseation, by fibrosis or by suppuration. Cold abscesses, fistulae and septic abscesses may supervene. Deposition of calcium salts, at first as a fine ' powdering' and later in more concrete masses, is a common degenerative manifestation, as in other forms of chronic tuberculous infection. Periadenitis is a sequela which leads to ' matting' of the nodes.

The pathological manifestations of tuberculous lymphadenitis show variations in different anatomical sites, but the classical histological picture is seen in the cervical nodes of children infected by the oro-pharyngeal route. In the mediastinum there is a peculiar proneness for the nodes at the tracheal bifurcation to become grossly enlarged during the course of measles and subsequently to become infected with the tubercle bacillus. In the omentum, tuberculous lymphadenitis may supervene without the development of tuberculous ulceration of the intestine. In early stages the nodes are enlarged and markedly inflamed, and, clinically, the more usual causes of acute abdominal symptoms may be simulated. In later stages calcification occurs.

Differential Diagnosis of Tuberculous Lymphadenitis. Whereas from the clinical viewpoint there may be some confusion as to whether an enlarged lymph node is due to tuberculosis, Hodgkin's dișease, sarcoidosis, lymphosarcoma or secondary lymphadenopathy, the histological picture is so characteristic that the diagnosis is readily established by biopsy examination of the node under consideration. Moreover the demonstration of Koch's bacillus in sections of the node is definitive. It is important to bear in mind that tuberculous infection of a lymph node may be associated with some other lymphadenopathy, either primary or secondary. Thus tuberculosis may co-exist in lymph nodes which are affected by Hodgkin's disease or by metastatic carcinoma.

(c) Syphilitic Lymphadenitis. Lymph node manifestations of syphilis are present in all stages of this specific chronic infection. These are essentially of a similar nature and are characterized by a great infiltration of the lymphadenoid parenchyma with plasma cells and lymphocytes. In nodes which are enlarged following upon a primary lesion, treponemes are demonstrable, either in the juice expressed from the node or in histological sections prepared by the methods of Levaditi or Bertorelli. In later stages fibrosis is a prominent feature. . In the gummatous stage necrosis is superadded without any caseation and small groups of multi-nucleated giant cells having centrally placed nuclei occur in considerable numbers. Important points of differentiation between gumma and tubercle comprise the presence of vascularity and the absence of caseation in the former. En- 


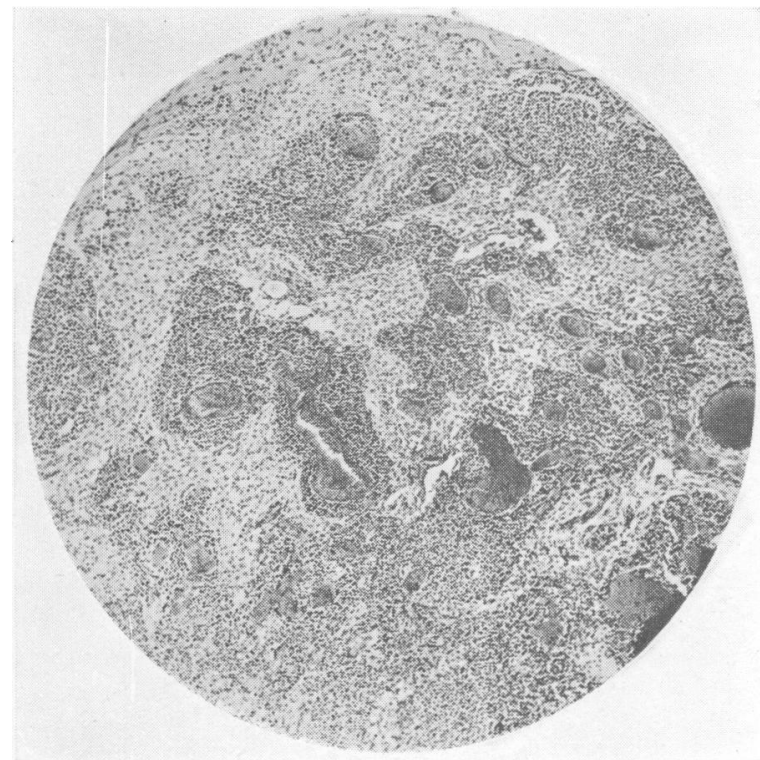

FIG. 1.-Reactionary sinus reticulosis.

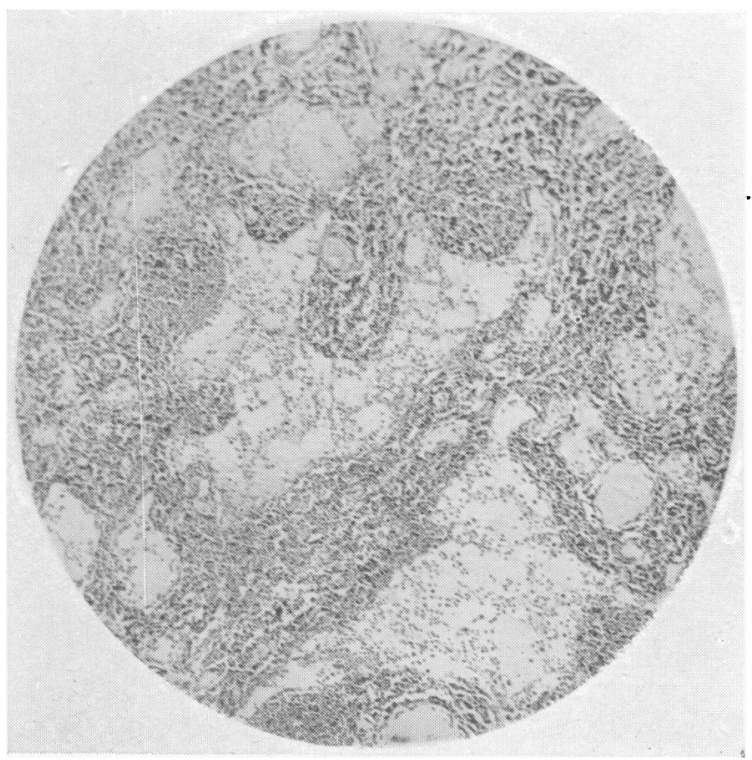

Fig. 3.-Sinus catarrh.

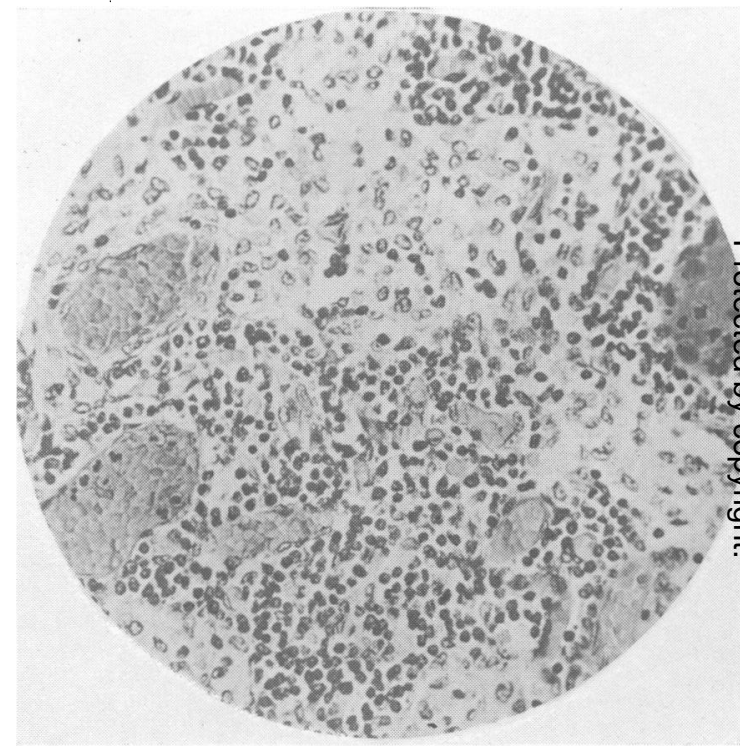

FIG. 2.-Reactionary sinus reticulosis. 


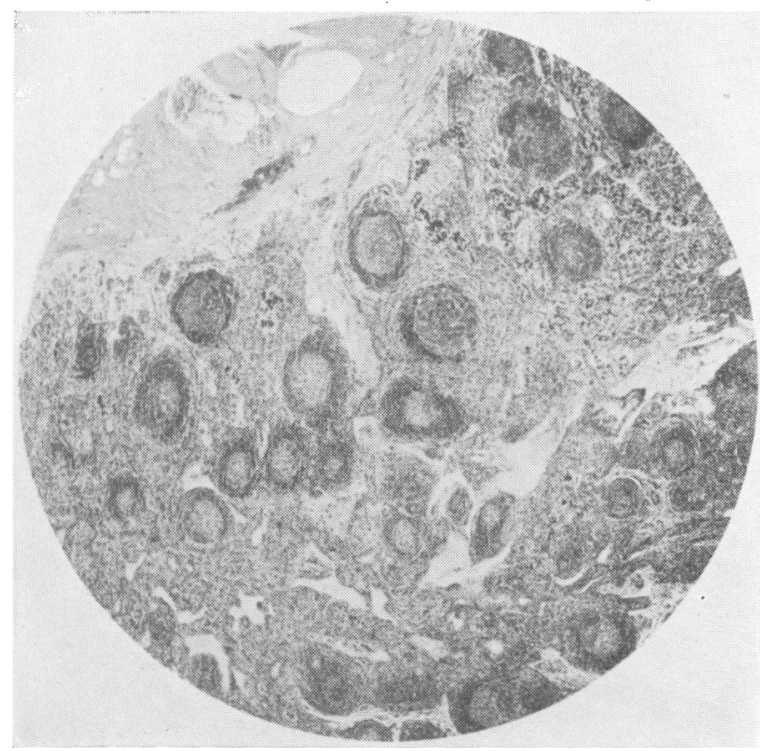

Fig. 4.-Reactionary follicular hyperplasia. Low power.

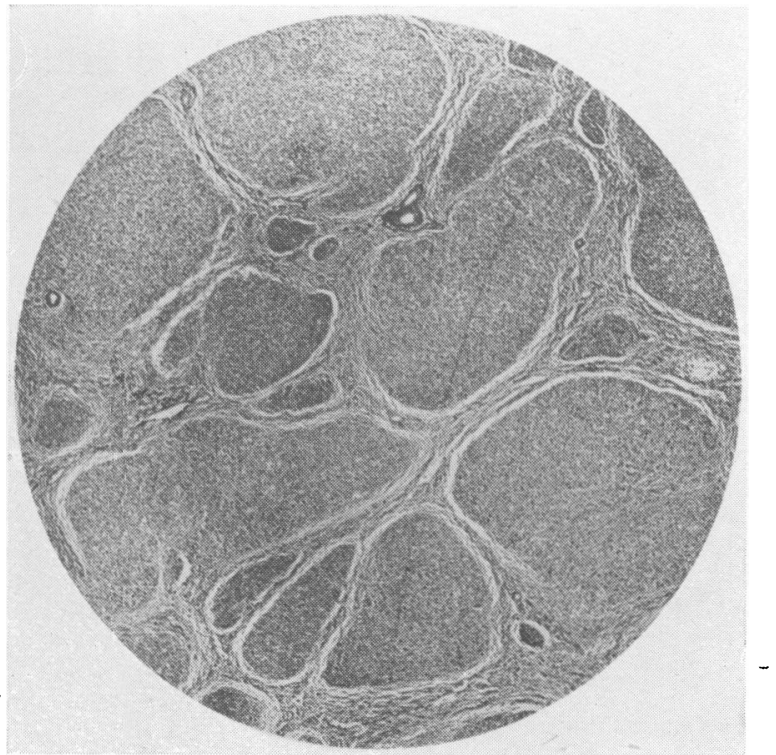

Fig. 6.- -Giant follicular lymphadenopathy or lymphoid follicular reticulosis.

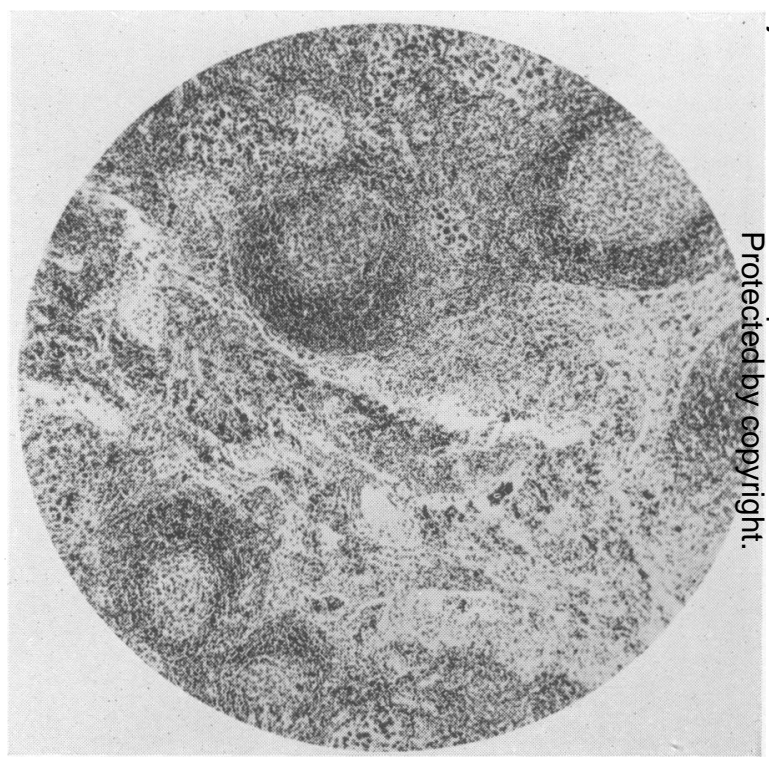

FIG. 5.-Reactionary follicular hyperplasia. High power.

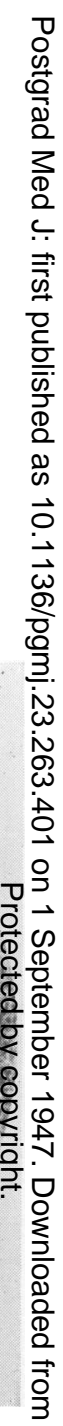

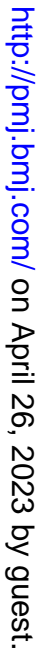



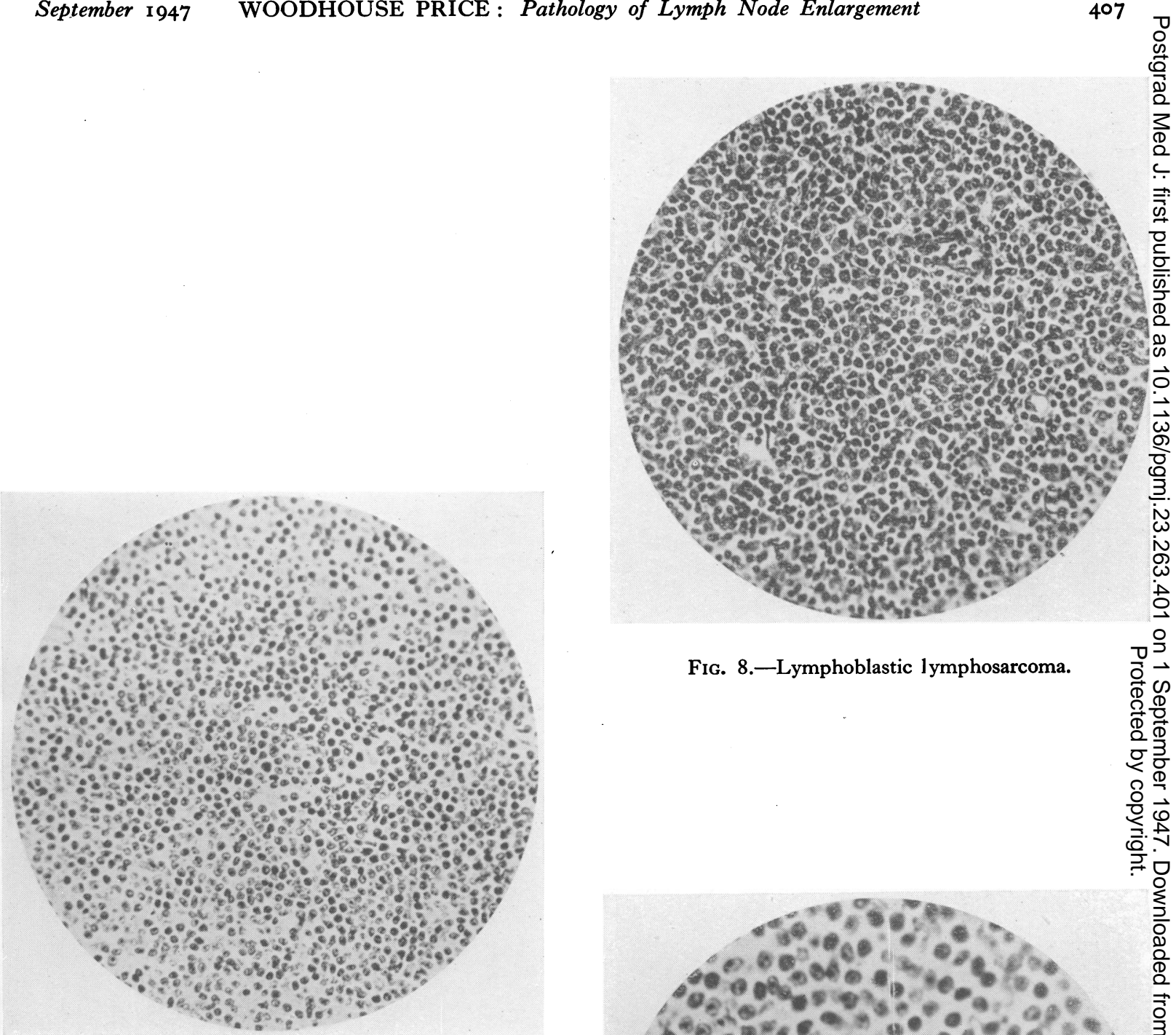

Fig. 8.-Lymphoblastic lymphosarcoma.

Fig. 7.-Lymphocytic lymphosarcoma.

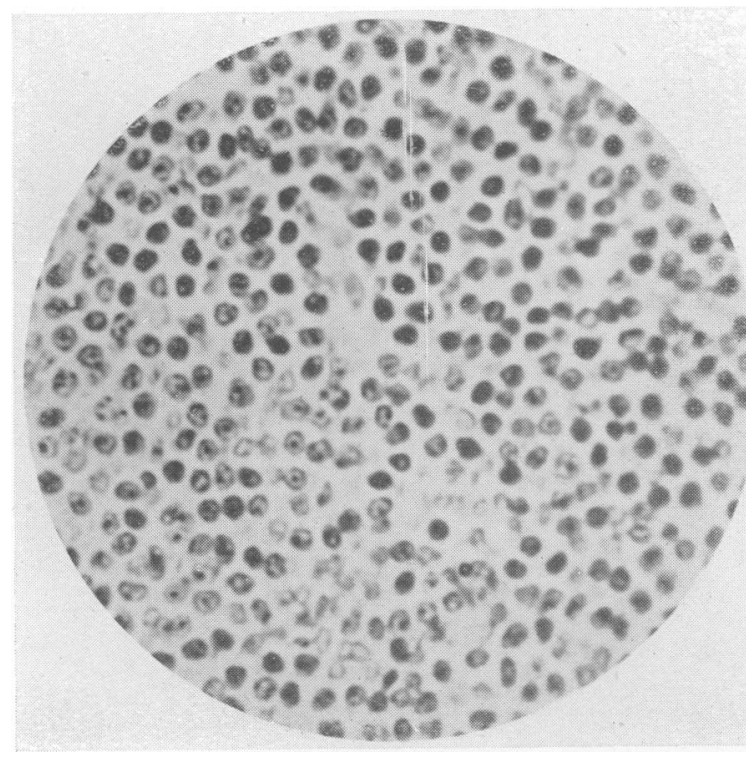

Fig, 9.-Reticulum-celled lymphosarcoma or reticulosarcoma. 


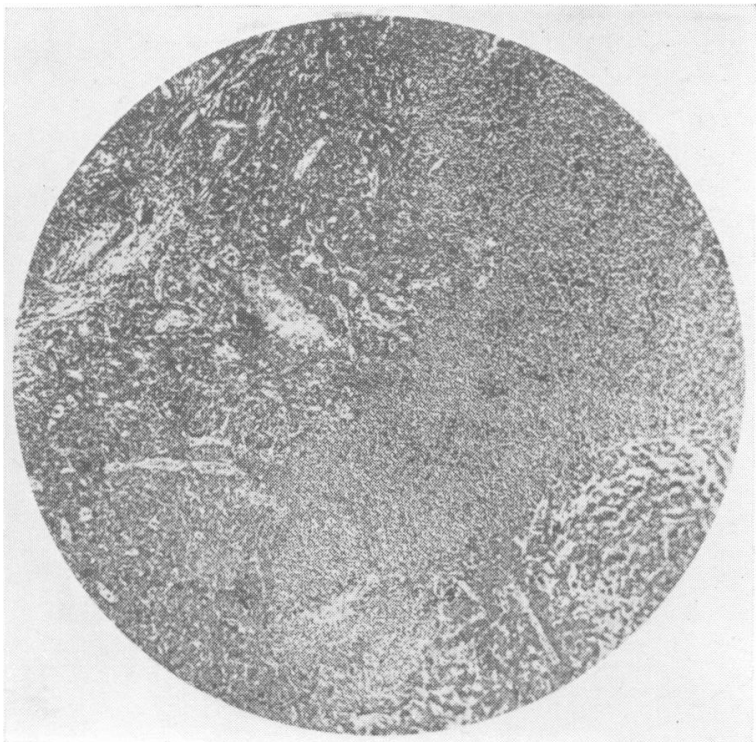

Fig. I0.-Leukaemic infiltration of the lymphadenoid parenchyma in early lymphatic leukaemia.

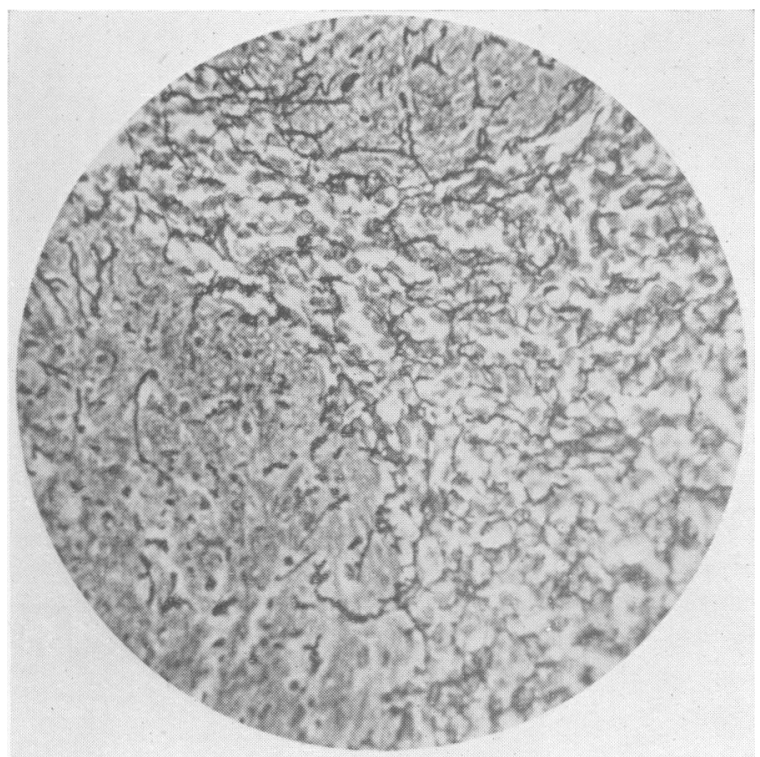

FIG. 12.-Lympho-epithelioma deposit stained to show reticulum.

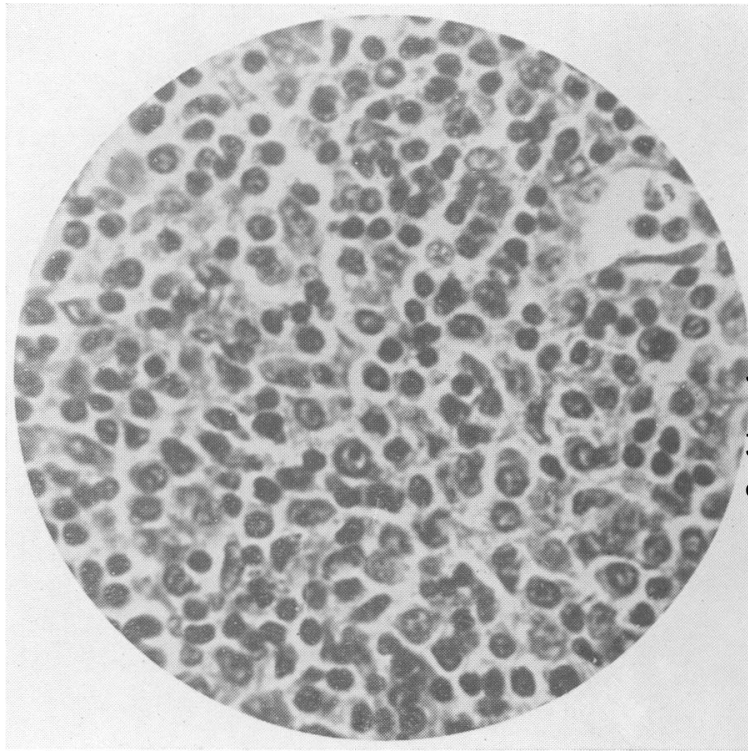

Fig. II.-Reticulosarcoma. High power. 


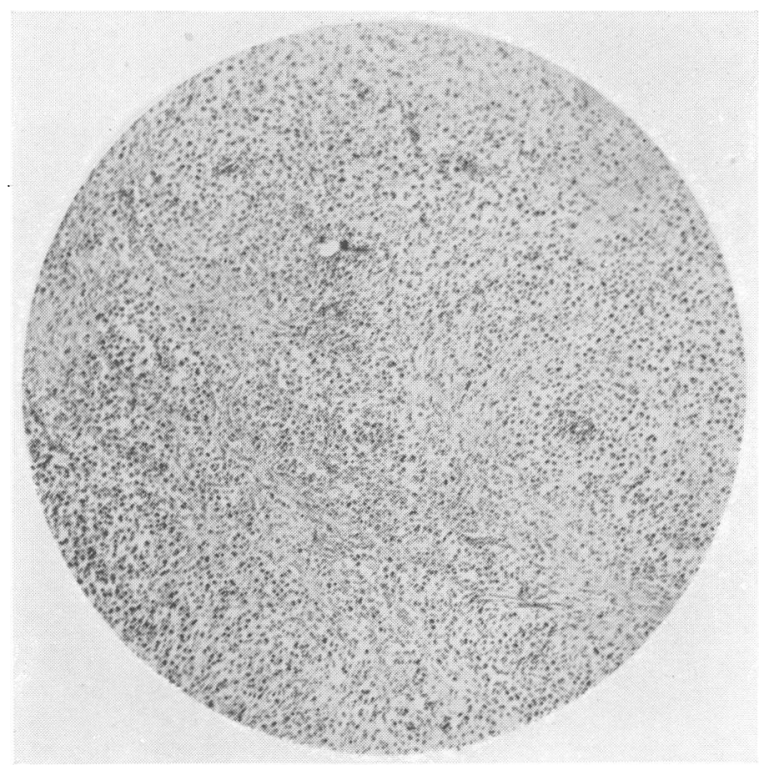

Fig. 13.-Hodgkin's disease. Lower power.

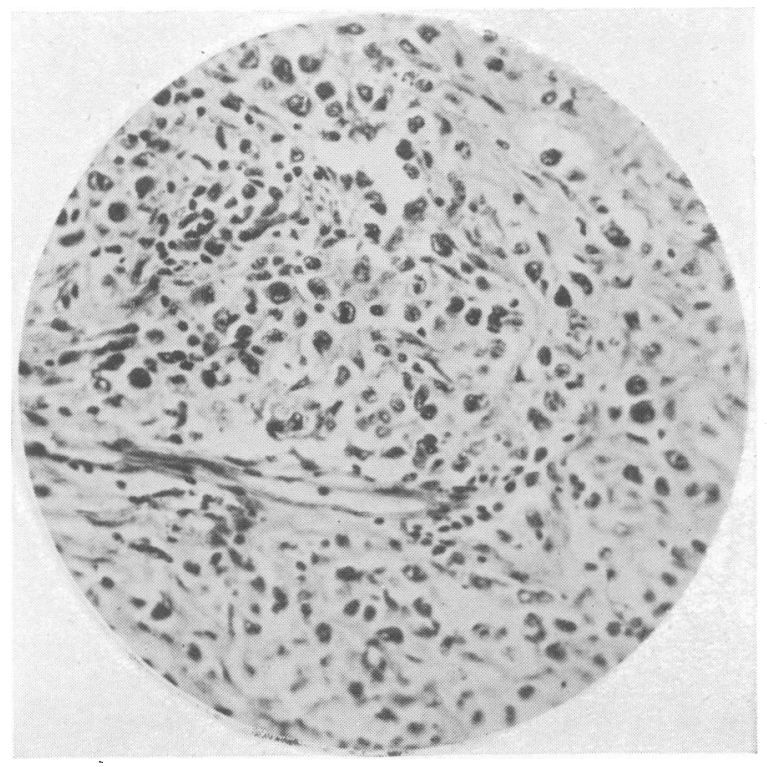

F1G. 14.-Hodgkin's disease. High power. 


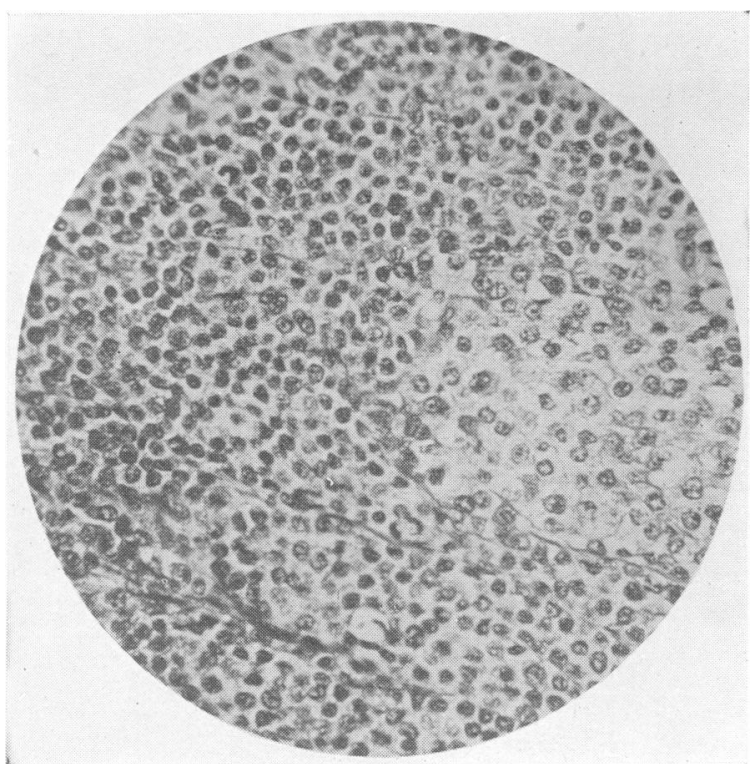

FIG. 15.-Deposit of achromic melanoma in a lymph node.

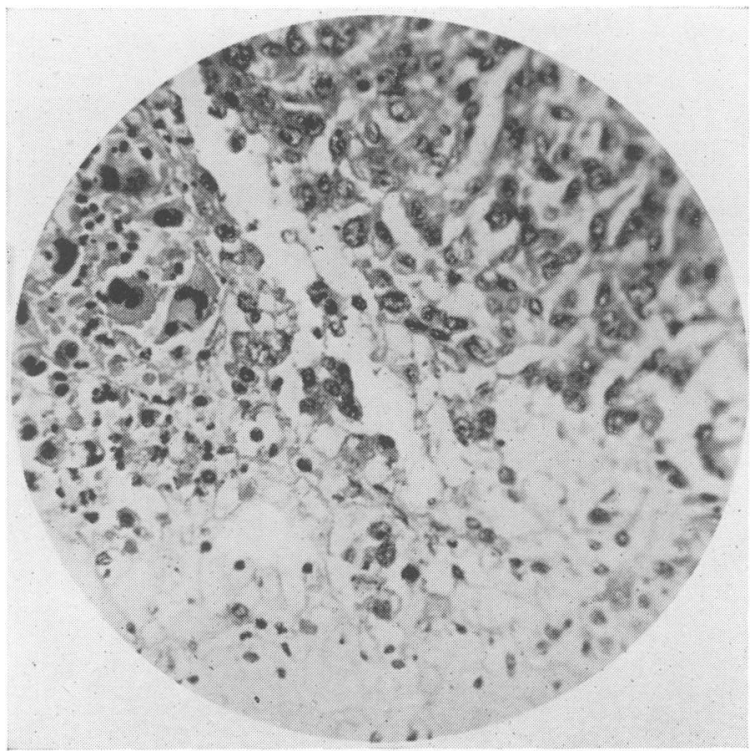

Fig. 16.-Deposit of melanoma in a lymph node. 


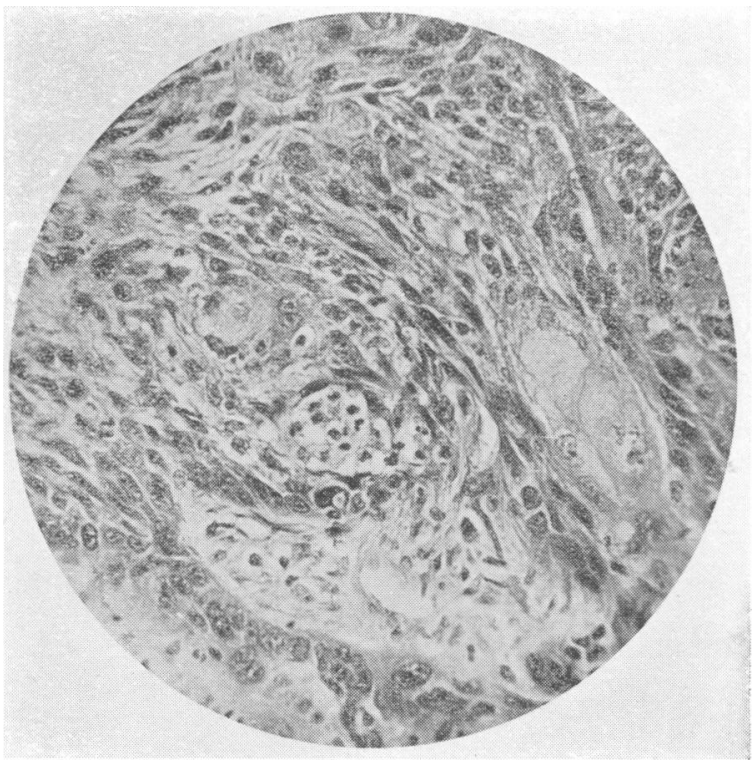

FIG. 17.-Lympho-epithelioma.

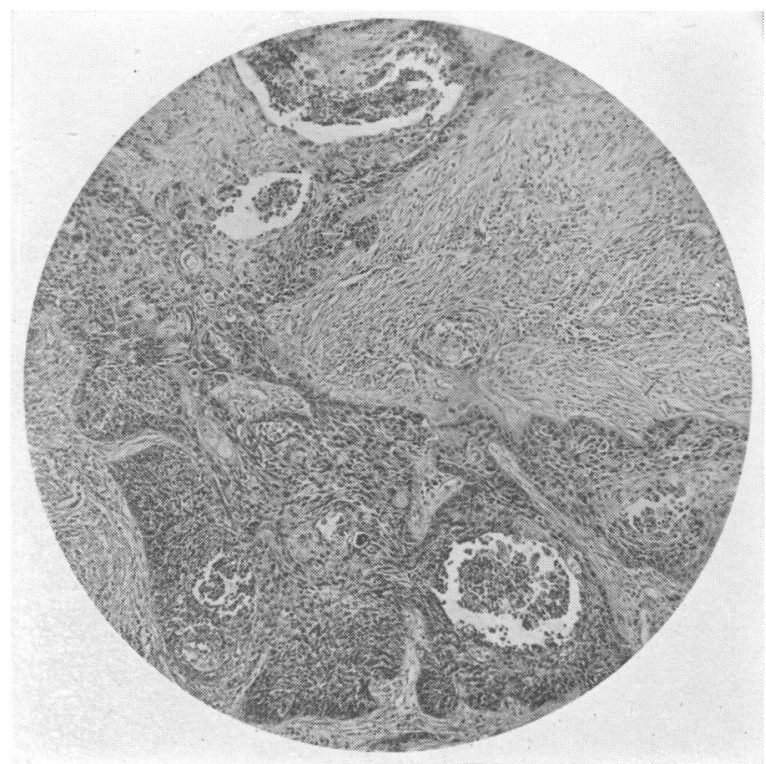

Fig. 18.-Lympho-epithelioma. 


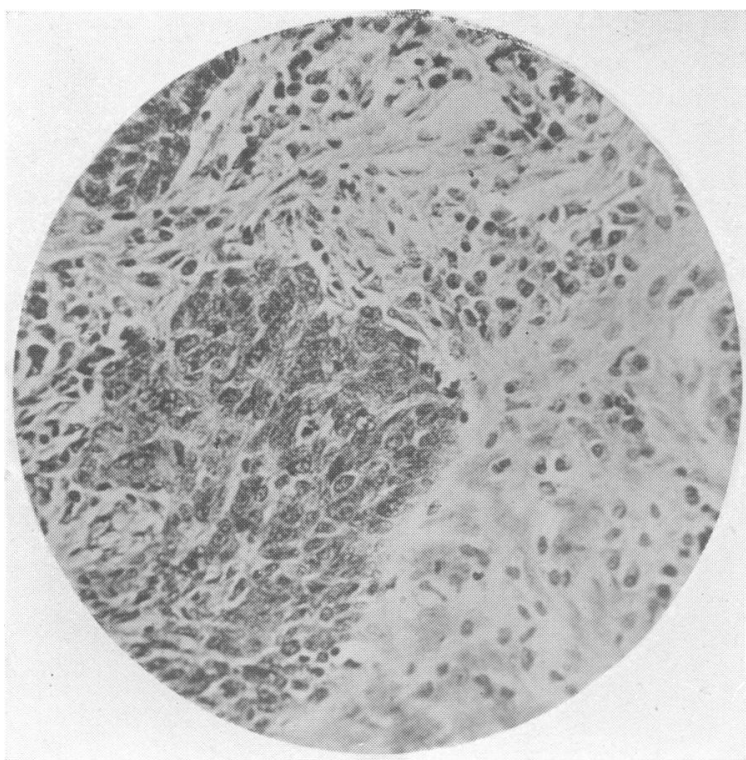

FIG. 19.-Lympho-epithelioma.

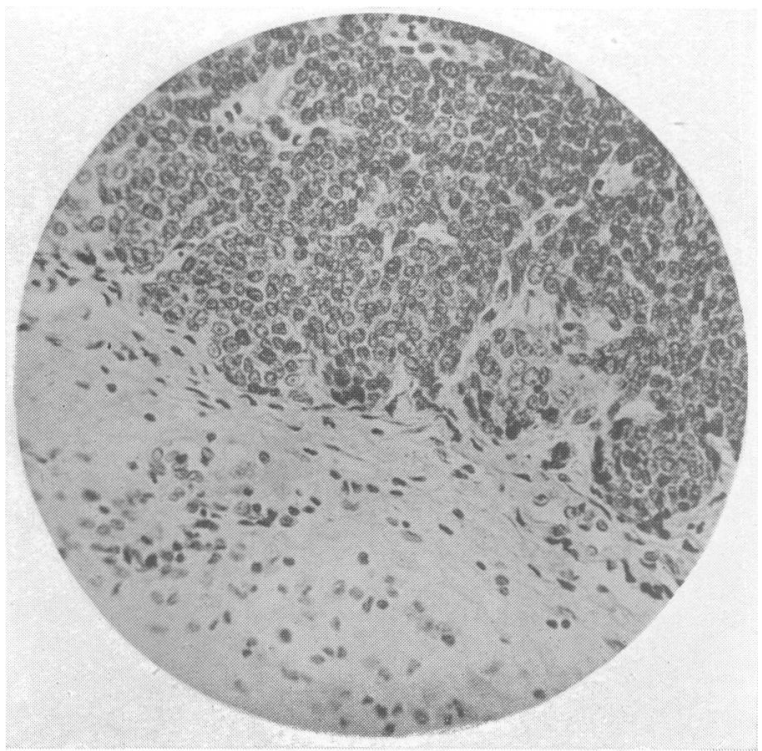


darteritis obliterans and periarteritis tend to be more marked in syphilis. The sites of predilection for gummatous involvement of lymph nodes are the epitrochlear and inguinal regions.

(d) Lymphadenitis in Lymphogranuloma Inguinale. In this chronic venereal infection which is caused by a filterable virus the lymph nodes affected show a difference of distribution in the two sexes. The inguino-cruro-iliac nodes are implicated in males while in females the lesion occurs in the pelvic and pararectal nodes. In females also stricture of the rectum may be a sequel. Histologically the nodes show marked periadenitis with the formation of multiple abscesses. The lymphadenoid parenchyma undergoes fibrosis and becomes diffusely infiltrated with plasma cells, lymphocytes and histiocytes, the histological picture being somewhat similar to that of some forms of chronic non-specific regional ileitis. Reticulo endothelial cells (' epithelioid cells') having a palisade distribution are found and the presence of a filterable virus is associated with gamma inclusion bodies. Diagnosis is established by the. Hofmann-Frei test, by which specific antigen is introduced intradermally.

It is to be noted that in another venereal infection, granuloma venereum, the causative agent is a Donovan body. Spread occurs from the skin and the lymphatic channels are not involved. The lesion responds to the intravenous administration of sodium antimony tartrate.

(e) Lymphadenitis in Acute Specific Fevers. Certain sites of predilection show enlarged lymph nodes in various infective conditions other than those already specifically described. In the exanthemata and in other acute specific fevers concomitant clinical signs serve to establish the diagnosis. Some of the principal lymph node enlargements in connection with such infective processes may be summarized as follows :-

Measles ; gross enlargement of the bronchial nodes which become liable to be infected subsequently with Koch's bacillus, complicated by cervical adenitis with suppuration.

Diphtheria; in faucial diphtheria the enlargement of the cervical nodes is proportionate to the extent of the membrane; in extra faucial diphtheria there may be enlargement of the inguinal nodes.

Scarlet fever; from the second to the fourth week there may be secondary adenitis in.. volving the nodes behind the angle of the mandible or under the insertion of the sterno. mastoid muscle.

Typhoid fever; there is hyperaemia and swelling of the mesenteric nodes. In Peyer's patches and in the solitary follicles of the ileum there is a pronounced reticulosis with swelling due to proliferation of reticulo endothelial cells.

Anthrax; a chain of enlarged painful nodes develops along the line of the lymphatic channels spreading from the 'malignant pustule.'

Glanders; this is a definite septicaemia which is diagnosed by means of the mallein test in animals or by recovery of bacillus mallei in six days after intraperitoneal inoculation of guinea-pigs. The tunica vaginalis of the testis becomes swollen; the cervical lymph nodes in man become enlarged and softened and may suppurate.

Plague; a tender bubo appears on the second or third day after infection; the commonest sites of predilection are the inguinal and femoral regions (70 per cent.), the axillary region ( 20 per. cent.) and the cervical and submaxillary regions (Io per cent.). The infected node may become the size of a hen's egg, and pain and suppuration are common. Diagnosis is established by aspiration biopsy when the characteristic bipolar bacillus is readily demonstrable on microscopic examination.

Malta fever; this is a bacteriaemia in which the mesenteric lymph nodes may become swollen but in which there is no ulceration of Peyer's patches. Bacillus melitensis may be recovered from swollen lymph nodes or by splenic puncture.

Glandular fever; this is a virus infection characterized by a mononucleosis of 40 per cent.-9o per cent., the total leucocytosis amounting to 12,000 to 30,000 per c.mm. blood. The upper cervical, mediastinal and mesenteric groups may be involved. There is 
a positive Paul-Bunnell reaction, as in serum sickness.

Serum sickness; enlarged lymph nodes, though rare findings may be associated with general oedema and albuminuria. The PaulBunnell reaction is positive and the common features are urticaria, swelling of joints, pruritis, mild pyrexia and malaise."

Tularaemia; infection of lymph nodes with swelling and suppuration followed by overlying ulceration occurs in keepers of rodents. The immediate cause is an insect bite. Clinically the condition is characterized by malaise and a prolonged inability to work.

Rubella; a tender adenitis affects principally the occipital, mastoid, posterior cervical, axillary and inguinal lymph nodes which remain discrete and firm:

Agranulocytic angina; Gangrenous and ulcerative stomatitis (Ludwig's angina) and also some cases of Vincent's angina may be followed by enlargement of the cervical lymph nodes. This is associated with a surrounding brawny induration, and a characteristic blood picture.

\section{Primary Lymphadenopathies}

The term ' lymphoma' was formerly applied indiscriminately when lymph nodes became enlarged from some endogenous cause not secondary to any exogenous primary focus of infection or secondary to neoplasm. This group is somewhat ill-defined and includes a variety of conditions of different nature of which the cause is often quite unknown. Broadly speaking the members of the group may be sub-divided into the reticuloses and the reticulosarcomata. The former subdivision, however, is complicated by the superimposition of lymph node manifestations of certain lipodystrophies.

The Reticuloses. The term reticulosis is applied to those changes in lymph nodes which are characterized by hyperplasia of the reticulo endothelial cells. The component cells of the lymphadenoid follicles and those of the sinuses may be affected independently or together. Follicular reticulosis is commonly associated with the presence of soluble toxins. Thus the regional lymph nodes become en- larged and show this change in infective processes such as tonsillitis, peptic ulceration or whitlow. Sinus or medullary reticulosis on the other hand is associated with particulate matter and is encountered in such conditions as bacteriaemia, pneumoconioses and neoplasm.

Follicular Reticulosis. Histological preparations of the enlarged lymph node shows markedly prominent lymphadenoid follicles. The reticulo endothelial ' germ centres' are hyperplastic and hypertrophic and also show a numerical increase. It is important to note that these hyperplastic follicles are sharply demarcated from the surrounding lymphadenoid parenchyma by a clearly defined peripheral zone of concentrically laminated lymphocytes. It is equally important that such follicles are not confused with small metastatic deposits of squamous or spheroidal celled carcinoma or other neoplasm.

Giant Follicular Lymphadenopathy. Synonym -lymphoid follicular reticulosis (RobbSmith). This is a special form of follicular reticulosis. It is a generalized enlargement of lymph nodes which may start focally and unilaterally but which may eventually involve the nodes of all regions. Clinically the lesion is characterized by a concomitant pleural effusion. The nodes affected are not grossly enlarged and the condition responds to radiotherapy. If left untreated, however, some form of lymphosarcoma is liable to develop.

When histological preparations are examined with the naked eye, or with the aid of a hand lens, the whole gland is seen to consist of rounded nodules which may reach a size up to $\mathrm{I} \mathrm{cm}$. in diameter. Microscopically these enlarged follicles present a characteristic appearance which is entirely different from that of the enlarged follicles in reactionary reticulosis. Whereas in the latter there is always a peripheral zone of concentrically laminated lymphocytes, in the former there is merely a narrow margin of fibroblastic and lymphadenoid stroma. When a single large follicle is observed under the microscope, it is seen to be composed of hyperplastic reticulo endothelial cells which show several mitotic figures of regular form. The lymphadenoid parenchyma and the sinuses are eventually 
entirely obliterated and the capsules show no gross pathological change. The picture as presented by the centre of an enlarged follicle is very similar to that of lymphosarcomanamely, a homogeneous field of lymphocytes, lymphoblasts or reticulo endothelial cells showing mitotic activity. Indeed it is not improbable that the lesion of giant follicular lymphadenopathy represents the earliest phase of development of multifocal lymphosarcoma.

Sinus Reticulosis. When the reticulo endothelial hyperplasia affects the cells of the subcapsular, communicating and medullary sinuses these channels become prominent owing to their distension with proliferating cells. It is important that the histological appearance of such distended sinuses is not confused with that of a diffuse metastatic infiltration of the sinuses with malignant epithelial cells.

Not infrequently the sinuses become distended with a clear hyaline oxyphile fluid exudate with or without proliferation of reticulo endothelial cells; such an appearance is due to oedema of the sinuses and is referred to as sinus catarrh. Peculiar forms of sinus reticulosis are met with as lymph node manifestations of defective lipoid metabolism which have been termed 'lipodystrophies,' 'lipoid histiocytosis,' 'lipoidoses' and 'storage reticuloses.' An account of the pathology of the principle members of this complex and ill-defined group is set forth under the general heading of 'sinus reticulosis' in order to clarify the essentially similar nature of multiple diseases which are clinically dissimilar.

The deposition and storage of specific types of lipoid substances consisting of cholesterol, cholesterol ester, cerebrosides and related compounds, in the reticulo endothelial cells and histiocytes of certain organs and tissues gives rise to a specific group of reticuloses in all of which the lymph nodes may become involved as a secondary manifestation of the disease in question. The organs primarily involved in the diseases under consideration include the skin, the subcutaneous tissues, the spleen, the liver, the bones and bone marrow, the uveal tract and the salivary glands.

Gaucher's disease. The histological manifestations result from a deposition and storage of a lipoprotein resembling a cerebroside. Splenomegaly occurs, accompanied by enlargement of the thoracic and abdominal lymph nodes. On hemisection the macroscopic appearance resembles ' cafe-au-lait' in colour. Microscopically the lymphadenoid parenchyma is infiltrated with the characteristic large Gaucher cells whose diameter may exceed $70 \mu$. The nuclei are small, nonreticulated and eccentric and the sytoplasm is copious and shows a wrinkled appearance.

Hypercholesterolaemia. Deposition and storage of cholesterol and of cholesterol ester occurs in a widely diverse group of cases, and histological evidence of the presence of these lipoids may be found in lymph nodes. The following clinically recognizable entities may be segregated :-

(a) Xanthelasma. This consists of a deposition of the lipoid in the histiocytes in the subconjunctival tissues of the eyelids and is not as a rule associated with enlarged lymph nodes.

(b) Xanthoma multiplex. In cases of biliary obstruction and in diabetes mellitus lipoids become distributed in the skin and are manifested as yellowish plaques.

(c) 'Xanthoma.' The term xanthoma does not imply a neoplasm of specific type, but has, by long usage, come to be applied to a peculiar type of lipodystrophy which develops as a secondary phenomenon in certain primary tumours in connection especially with tendon sheaths, joint capsules and bones. The tumour may be a fibrosarcoma, fibroma, giant celled tumour of the osteoclastoma type, or certain forms of osteogenic sarcoma. The characteristic foamy cells of this form of xanthomatous degeneration may be found in metastatic deposits in the regional lymph nodes.

(d) In the Hand-Schuller-Christian syndrome, cholesterol and cholesterol ester are deposited in endothelial cells of capillaries and in histiocytes. The lipoid becomes liberated into the tissue spaces which results in the formation of granulation tissue and fibrosis as a reactionary phenomenon. The meninges become involved with firm putty like deposits of yellowish appearance which encroach upon the bones of the skull, leading to decalcification. This is a manifestation of skeletal lipoidal granulomatosis. 
(e) Niemann-Pick disease. This rare disease of childhood, occurring in the first year or two of life and terminating fatally at an early age, is due to storage of phosphatide lipoid or a mixture of cholesterol and phosphatide. The lymph nodes become enlarged and of yellowish colour. Microscopically they contain characteristic cells of about $35 \mu$ in diameter in which the cytoplasm is finely vacuolated.

(f) Warren-Tay-Sach's disease. The lesion in amaurotic family idiocy is a storage of cerebroside protein.

Sarcoidosis. Synonyms-Stengel-Wolbach sclerosis; chronic medullary reticulosis of Robb-Smith ; endothelial tuberculosis.

This peculiar form of chronic sinus reticulosis is shown pathologically by the infiltration with pale lipoid containing cells sometimes associated with multinucleated giant cells resembling those of tuberculosis. In consequence the lymph nodes become enlarged and are clinically associated with lesions of the uveoparotid tract, osteoporosis of the digital phalanges and Boeck's dermal sarcoid. Essentially, the lymph node enlargement is due to an infiltration of the lymphadenoid sinuses with reticulo endothelial cells which have undergone a lipodystrophy. In early phases of this process the affected node reveals small groups of pale staining large ovoid cells whose cytoplasm has a parboiled appearance and whose nuclei are small and eccentric. In later phases these small groups tend to become confluent and to form lobulated masses which occupy a large proportion of the lymph node. A certain amount of normal lymph adenoid parenchyma generally remains and the distinction between sinuses, follicles and parenchyma persists to a greater or less degree. There are two important points of distinction between sarcoidosis and tuberculosis; in the former caseation does not occur and no tubercle bacilli are demonstrable.

There are diverse manifestations of sarcoidosis, and, while the essential lesion is similar in all instances, confusion has been engendered on account of the diversity of nomenclature which has been employed in relation to lesions in various tissues and organs. The following main types have been segre- gated as pathological entities, though in reality they are merely different manifestations of a single pathological process :-

(a) The dermal sarcoid of Besnier-Boeck. This is an infiltration of the epidermis with lipoid containing reticulo endothelial cells which, through long standing custom, are usually referred to as ' epithelioid cells.'

(b) The subcutaneous sarcoid of DarierRoussy. This is the counterpart of the dermal sarcoid in the cutis vera and superficial fascia.

(c) Stengel-Wolbach sclerosis. This is a chronic form of reticulosis affecting primarily the sinuses of lymph nodes. The subcapsular sinuses first, and eventually the communicating and medullary sinuses become grossly distended with the characteristic cells of the lesion. An important point in differentiation is the essential retention of normal lymphadenoid architecture ; this is modified only in relation to the extent of the sinus infiltration.

(d) Osteitis multiplex of Jungling. This is a manifestation of sarcoidosis affecting principally the digital phalanges of the fingers and toes, but sometimes affecting other bones.

(e) Uveo-parotitis of Heerfordt. In this manifestation the uveal tract, lachrymal glands and salivary glands are involved.

(f) Endothelial tuberculosis of Zinsser. This name was formerly applied to the sinus lesions of lymph nodes which are now recognized as the essential lesion of sarcoidosis.

Erythrodermia. This chronic skin lesion which was formerly termed exfoliative dermatitis, is a peculiar form of reticulosis of the skin characterized by the presence of a lipomelanic reticulosis in the associated lymph nodes.

\section{Hodgkin's Disease}

Synonyms-Lymphadenoma ; fibromyeloid reticulosis of Robb-Smith.

This form of primary lymphadenopathy whose aetiology is unknown begins in a single lymph node and soon spreads to adjacent members of a group. A little later there is bilateral involvement and eventually generalization may occur with involvement of the 
cervical, axillary, mediastinal, abdominal and inguino crural groups.

Macroscopically the affected nodes may be grossly enlarged but they tend to remain discrete. They are of firm consistency and pale appearance. On hemisection the cut surface gives the impression of being somewhat fibrous ; there may be patches of necrobiosis but there is no caseation. Microscopically the histological picture shows considerable variations, but there are certain classical features which may be represented in varying proportions in different cases. Characteristically the essential features may be enumerated under five headings:-pleomorphism, loss of lymphadenoid architecture, fibrosis, infiltration with eosinophilous cells, and the presence of Sternberg-Reed giant cells. Each of these criteria requires amplification.

Pleomorphism. The characteristic picture of a well-developed lesion of Hodgkin's disease may be modified if a lymph node is examined at a very early or a very advanced stage of its evolution. In the early stage a common feature is a diffuse interstitial infiltration of the lymphadenoid parenchyma with reticulo endothelial cells. The heterogeneous morphology of the node serves to distinguish the lesion from recticulo sarcoma, in which homogeneity is the keynote. This cellular proliferation may be accompanied by an occasional eosinophilous cell and an occasional Sternberg-Reed giant cell, but there may be relatively slight fibrosis. In an advanced stage in the evolution of the lesion fibrosis may be the predominant feature. In the more virulent forms of the disease (which have been designated malignant Hodgkin's disease, or Hodgkin's sarcoma) certain features may be markedly exaggerated. In some cases the Sternberg-Reed cells may be abnormally large and hyperchromic and excessively numerous; in other cases eosinophilous cells predominate.

Loss of lymphadenoid architecture. Except in early phases of the development of this disease there is total obliteration of the lymphadenoid sinuses and follicles and considerable thinning out of the lymphadenoid parenchyma. The whole node becomes eventually completely replaced by the peculiar tissue characteristic of Hodgkin's disease.
Fibrosis. Characteristically this occurs in the form of small localized tufts of collagen of some thickness and there is a marked tendency for this fibrous tissue to undergo hyaline degeneration. The distribution is well shown by the application of methods for reticulum staining.

Eosinophilous cells. These are present in very variable numbers in different cases of the disease, but generally they are not excessive. In some instances an eosinophilia is shown in the blood picture. Though these cells are characteristically present in most lymph nodes affected by the lesion of Hodgkin's disease, they are not per se pathognomonic of the disease and are quite frequently observed in other forms of lymphadenopathy. Nevertheless, their presence in a node which shows little change of morphology other than slight fibrosis and a general thinning out of the lymphadenoid architecture, provides a criterion in favour of the diagnosis of Hodgkin's disease.

Sternberg-Reed giant cells. These are also known as ' mirror image giant cells' from the symmetrical distribution of their nuclei which are generally four in number; the appearance of these cells has been likened to that of pennies on a plate. The cell is of circular or ovoid form and characteristically contains four nuclei whose margins to some extent overlap and which are symmetrically arranged in the abundant hyperchromic cytoplasm. Such cells are sometimes present in very scanty numbers, but they tend to be excessively numerous and of bizarre form in the more malignant types of the disease.

Reticulo endothelial cells. The presence of these cells out of their normal environment may be cited as an additional criterion to the five already described. They are seen typically in the earlier phases of development of the disease but may be insignificant when the lesion is more advanced. The normal distribution in the follicles and sinuses is replaced, in early stages of the disease, by a diffuse infiltration throughout the attenuated lymphadenoid parenchyma.

Morbid anatomy of Hodgkin's disease. In cases which come to necropsy, enlarged lymph nodes showing the characteristic macroscopic 
and microscopic features already described may be found in any region, and polyserosites is usually present. In advanced cases the appearances of the lesions are those of a malignant neoplasm. There may be contiguous extension to bones, and deposits may be found in the lungs, liver, spleen and other internal organs. To the naked eye these deposits generally appear as multiple white nodules of ill defined outline, less sharply demarcated than metastatic deposits of carcinoma, but resembling the focal deposits sometimes found in leukaemias.

\section{Lymphosarcoma}

This is a primary malignant affection of the lymph adenoid system which shows a variety of pathological manifestations. Macroscopically the affected nodes are grossly enlarged, sometimes to the size of a hen's egg. They are of soft consistency and almost white appearance. On hemisection the cut surface appears homogeneous and opaque or semitranslucent. This uniformity contrasts sharply with that of lymph nodes which are the site of metastatic carcinoma. In the latter the cut surface of the hemisected node appears nodular, especially after a short period in fixative solution. The macroscopic appearance is also readily distinguishable from that of Hodgkin's disease in which fibrosis plays so prominent a part.

The manifestations of lymphosarcoma may be limited to the nodes of a local region such as the neck or the abdomen, but in advanced cases generalization is the rule, with deposits in the viscera.

Microscopically the histological picture presents a remarkable degree of homogeneity with complete loss of differentiation of the normal components of the lymph node. The microscope reveals a uniform field of lymphocytes, lymphoblasts or reticulo endothelial cells. In consequence of this selective cytology three distinct types of lymphosarcoma are distinguishable according to the nature of the predominant cell. These are termed respectively lymphocytic lymphosarcoma, lymphoblastic lymphosarcoma, and reticulum celled lymphosarcoma; the latter has the alternative name of reticulo sarcoma.

The morbid anatomy of this disease is variable. In some cases internal organs such as the spleen, liver and kidneys show circumscribed nodules of lymphosarcoma which are essentially metastatic deposits. In other cases the spleen may be one of the primary sites of this form of primary lymphadenopathy. A difficulty that not infrequently arises in the differential diagnosis of an excised lymph node for biopsy purposes is the distinction between lymphosarcoma and aleukaemic lymphatic leukaemia. This point is given consideration in connection with the haematopoietic affection.

Primary lymphosarcoma, in which lymph node manifestations are inconspicuous, may arise in the lymphadenoid tissues of the thyroid gland. Another primary form is manifested as a thymoma and is associated with the clinical signs of myasthenia.

\section{Lymphatic Leukaemia}

This disease, in view of our imperfect knowledge of its aetiological relationships, may be regarded provisionally as a sarcoma of the blood and blood forming organs as the primary affection, but the lymphadenoid system is not infrequently implicated. In the usual type of case the diagnosis is readily arrived at from an examination of the blood picture. If, in such a case, there is involvement of lymph nodes and a node is excised for biopsy purposes, no difficulty is presented to the histologist, because the microscopic preparation reveals the presence of excessive lymphocytes or lymphoblasts in the blood vessels within the lymph node and in those permeating the adjacent fibro fatty tissue.

On the other hand, in cases of aleukaemic lymphatic leukaemia, difficulty may arise in histological interpretation owing to the fact that, superficially, the microscopic appearances of lymph nodes in leukaemia are almost identical with those of lymphosarcoma. There are, however, certain special features which serve to differentiate the two conditions. Sometimes the pathologist is fortunate in having for examination a lymph node in which 
some residue of normal architecture persists. In such a case, a leukaemic infiltration of the lymphadenoid parenchyma, precisely similar to that seen in the renal parenchyma in leukaemia, is perceptible. Another point is that the adjacent fibro fatty tissue may show a leukaemic infiltration, especially in those nodes whose morphology is of the reniform or Cshaped type. Finally, in lymphosarcoma mitotic figures are unduly prominent and examples of heterotype mitoses may be encountered.

\section{Secondary Lymphadenopathies}

Lymph nodes often become enlarged owing to the presence of metastatic deposits of carcinoma and other malignant tumours; such enlargements may be conveniently referred to as secondary lymphadenopathies, but it must be borne in mind that metastatic deposits may occur in lymph nodes without enlargement.

It is important in this connection that full recognition be given to the fact that the degree or extent of metastasis does not necessarily bear any proportionate relationship to the macroscopic size of the lymph node enlargement. In order to illustrate this point a description of the findings on pathological investigation of a typical surgical specimen will suffice. When radical mastectomy has been performed for mammary carcinoma it is desirable that the degree and precise mode of spread of the malignant process be ascertained as far as possible from a detailed examination of the specimen. To this end, a routine procedure has been found to be of assistance and its essentials are set forth hereunder.

After a careful description of the position, size and shape of the primary tumour in the breast, the axillary contents are dissected and the number, size and relative positions of the lymph nodes noted. In addition, the skin overlying the tumour and the underlying pectoral fascia and pectoral muscle are searched for possible secondary or contiguous deposits. Representative portions of tissue are selected for microscopic examination from the primary tumour, from the skin, pectoral fascia, pectoral muscle, from the neighbouring tissue of the corpus mammae and from the axillary fibro fatty tissue. In addition, all lymph nodes found on dissection are prepared for microscopic examination.

It is a matter of frequent experience that the axillary lymph nodes show a considerable range in size from 2 or 3 millimetres up to 2 or 3 centimetres. It is important to realize that a solitary metastatic deposit of carcinoma mammae may be present in one of the smallest nodes while the larger nodes remain unaffected by the malignant process. Histological findings such as these serve to emphasize that, except in cases of gross malignant involvement of lymph nodes, the 'staging' of breast carcinoma is impossible on clinical grounds alone.

\section{Mode of development of lymph node meta-} stases. Dissemination of malignant cells from a primary tumour occurs in three ways-by direct intercellular extension into neighbouring tissues, by lymphatic permeation and embolism, and by vascular embolism. To these may be added the rarer method of retrograde embolism. Hence a lymph node may become inseminated with malignant cells by contiguous extension, by way of the afferent lymphatic channels around the periphery, or by way of the afferent arteriole centrally. Realization of these anatomical principles serves to explain the diverse histological appearances of lymph nodes which are the seat of metastasis. In some instances histological preparations show afferent lymphatic channels in the fibro fatty tissue immediately adjacent to a lymph node, distended with plugs of malignant epithelial cells. The subcapsular lymphatic sinus may also be seen to be distended with such cells. When some time has elapsed the 'malignant seedling' as it may appropriately be called, grows at the expense of the lymphadenoid matrix in which it is deposited and the normal architecture of the lymph node becomes unrecognizable owing to the almost complete replacement of the lymphadenoid parenchyma by malignant tissue. In examples where malignant dissemination has been haematogenous the earliest phase in the evolution of a metastatic deposit is in the centre of the node where the afferent arteriole undergoes poly. chotomy. 
In attempting prognostication in a given pathological investigation the relative significance of lymphogenous and haematogenous deposits in lymph nodes must be given due consideration. It is self evident that the latter most probably indicate haematogenous deposits in bones or in the internal organs.

Relation of malignant parenchyma to stroma. There is not always a strict parallelism between the histological appearances of a primary tumour and those of its metastatic deposits. The primary carcinoma in a breast, for example, may be mainly of scirrhous morphology while the lymph node metastases may be highly cellular, of medullary type or showing an alveolar form, and having no appreciable stroma. In other instances the stroma is a predominating feature in the metastases while the primary tumour may have shown a medullary morphology. Such distinctions, however, are relative and not absolute, for many tumours tend to be heterogeneous rather than homogeneous, and this is especially true of many carcinomata.

Although the stroma may be regarded as an essential part of the tumour and not merely a cellular reaction on the part of the host, it is evident from the foregoing considerations that no conclusions can be drawn with certainty as to the precise histological class to which a primary tumour belongs from an examination of an isolated lymph node metastasis. When confronted with a lymph node which proves on histological examination to contain a metastatic deposit of neoplasm the main concern of the pathologist is to differentiate the various types of primary tumour presented in the metastatic deposit and to assist his clinical colleagues in the localization and essential nature of a dormant primary tumour.

The phenomenon of squamous metaplasia. It is well recognized that epithelium which was originally columnar, cubical or transitional in character, may undergo squamous metaplasia, which implies a change of morphological configuration. Such a transformation proves not infrequently to be a predisposing factor in the development of squamous carcinoma. It is at once apparent that, in cases where carcinoma of squamous type develops as a result of metaplasia of epithelium which was primarily columnar, cubical or transitional, an examination of a lymph node metastasis will be of no avail in assessing the specific nature of the epithelial cells from which such a tumour primarily arose, unless there are clinical indications of the presence of a primary tumour in a specific organ or tissue.

Squamous metaplasia tends to occur in connection with branchial fistulae and cysts, in persistent remnants of the thyroglossal duct, in the gall bladder and biliary passages, and in the respiratory and urinary tracts. Recognition of such an eventuality may serve to explain the development of certain otherwise inexplicable metastatic deposits.

It is thus possible to account for the development of well differentiated squamous carcinoma, with keratinization, epithelial. pearl formation and intercellular protoplasmic bridges in such primary sites as the thyroid gland, the bronchus, the gall bladder, bile ducts, renal pelvis, ureters and . urinary bladder.

Another phenomenon, which may appropriately be termed 'secondary squamous metaplasia,' occurs after the development of a primary adenocarcinoma in a glandular organ. This is more readily detected owing to the heterogeneous morphology of the tumour, which may be applicable to both primary and metastatic tumours. On the other hand, difficulty in interpretation may again arise if the material available for microscopical examination comprises a lymph node containing a metastatic deposit which is entirely squamous in its morphology. The reverse phenomenon of columnar metaplasia has not been encountered.

\section{The Differential Diagnosis of Specific}

\section{Cases of Lymph Node Enlargement}

Sometimes the pathological nature of an enlarged lymph node is self evident from clinical considerations and from its regional distribution, while other cases demand the application of a number of histopathological tests of a specific nature.

The initial point to be decided is whether the enlargement of a lymph node is inflam- 
matory, neoplastic or reactionary. If inflammatory, is it acute inflammation, chronic inflammation or a chronic infective granulomatous lesion? If neoplastic, is it primary or metastatic? If reactionary, is it of a simple nature or associated with some form of lipodystrophy?

After many of the predisposing factors which result in lymph node enlargement have been excluded by clinical tests, supplemented by serological, haematological and bacteriological investigations, there still remains a considerable group which is dependent upon histological diagnosis alone for diagnostic differentiation. This affirmation is applicable especially to those cases of lymph node enlargement which have been shown to be of a neoplastic nature, but in which there is no clinical indication as to the nature of the tumour or of its primary site.

Differential histological diagnosis is called for specifically where the primary tumour is quiescent or concealed. There are many instances where the first manifestation of a neoplasm is lymph node enlargement due to metastasis. Histological differentiation of such a deposit requires a co-ordination of the histological findings with the anatomical region involved. The development of metastases in certain regional nodes is a useful pointer in some instances. For example, when the inguinal nodes are involved, the finding of adenocarcinoma on biopsy examination suggests a primary tumour in the colon or rectum rather than in the stomach, while a deposit of similar nature in the cervical region suggests a tumour in the stomach or in the naso-pharynx. It must be emphasized however that too much dependence must not be placed on the regional distribution of the affected nodes, and that every effort should be made on the part of the pathologist to arrive at a diagnosis purely from histological considerations.

In some instances, histological differentiation, in the absence of clinical signs and symptoms, affords the only means of assessing the site and nature of the primary tumour. An enlarged supraclavicular node serves as an example. Metastasis in such a node may be secondary to a carcinoma in a large number of possible primary sites which include the pharynx, larynx, oesophagus, thyroid, skin of the trunk or upper extremity, gastrointestinal tract, hypernephroma, malignant melanoma, malignant ovarian cystadenoma, plasmacytoma, myeloma, seminoma, sialoma, and so on.

Histological differentiation is sometimes dependent upon the application of specific staining reactions to microscopical preparations. Brief notes are appended hereunder to aid in the differentiation of a number of specific forms of primary neoplasm whose local manifestation may be encountered in lymph nodes.

Lympho epithelioma. This variety of anaplastic squamous carcinoma arises in the special lymph epithelium of the tonsils and nasopharynx and may also be found in the palate and oropharynx. Histologically the lesion is characterized by a malignant hyperplasia of squamous epithelium in direct contact with a lymphadenoid stroma. Two forms are recognized - the Schmincke type which is deeply infiltrating and which is more commonly met with in the tonsils, and the Regaud type which is lobulated and shows an argyrophile reticulum of special distribution, and which is more common in the nasopharynx.

The first clinical manifestation of lympho epithelioma is usually an enlarged cervical lymph node due to the presence of a metastatic deposit. A peculiar clinical feature is the latency of the primary tumour. Even bulky lymph node metastases may appear before the small primary neoplasm evokes symptoms. This tumour is recognized as being particularly radio sensitive.

Squamous carcinoma. In well differentiated types with keratinization and epithelial pearl formation there is little danger of confusing the growth with any other types of tumour. The only difficulty that arises is when the tumour results from squamous metaplasia in epithelium of different ontogeny. In the more dedifferentiated or anaplastic forms of primary squamous carcinoma a useful aid to the differentiation of the type of growth is the accentuation of intercellular protoplasmic bridges by special staining methods. If 'prickle cells' are demonstrable the presence of squamous carcinoma is established. 
The finding of a metastatic deposit of squamous carcinoma in a lymph node is an indication, in the first instance, for a careful clinical search for a primary squamous carcinoma in the region most probably concerned. The primary tumour might well be disclosed in the tongue, respiratory tract, renal tract or external genitalia. Lympho epithelioma must not be ignored as an alternative possibility when the cervical nodes are involved.

The phenomenon of squamous metaplasia may cause considerable difficulty if its possibility is not borne in mind. It occasionally happens that the primary tumour in a glandular organ such as the breast or the mucosa of the gastrointestinal tract, undergoes squamous metaplasia. Such a phenomenon would be readily recognizable from a microscopic examination of the primary tumour, but the difficulty is encountered when the lymph node deposit presents entirely squamous characters.

Adenocarcinoma. In well differentiated types of adenocarcinoma a distinct alveolar or papilliform morphology is discernible and it only remains to determine the nature of the epithelium from which the primary tumour probably arose. In some instances the differentiation presents little difficulty while in other instances the precise nature of the growth may be obscure.

The first step in differentiation is the application of a staining reaction for mucin. If the reaction of the tumour cells to mucicarmine is strongly positive this is an indication of a primary malignant lesion in the gastrointestinal or biliary tracts. If the mucicarmine reaction is faintly positive it indicated a primary tumour of the ovaries or endometrium. A negative mucicarmine reaction is suggestive of an anaplastic squamous carcinoma.

Further differentiation is based on morphological considerations. The stomach and small intestine develop a type of adenocarcinoma in which the cytoplasm of the malignant epithelial cells is comparatively clear and in which nuclear reduplication is not marked. The colon and rectum, on the other hand, develop tumours composed of tall columnar hyperchromic cells, usually with strongly basophile staining, in which nuclear reduplication is so marked that the cytoplasm is almost or completely filled with supernumary nuclei.

A point of distinction between carcinoma of the caecum and that of the rest of the colon and rectum is that in the former a marked oxyphil staining reaction of the cytoplasm is sometimes discernible and the morphology of the malignant alveoli is markedly cribriform.

Ovarian tumours are characterized by having a pyriform morphology. Examination of a papilliform process of such a tumour as a malignant ovarian cystadenoma shows that the outline of the papilla is not smooth and regular but somewhat nodular; the individual cells are pear shaped, being attached at the base by a narrow pedicle. Moreover, ovarian tumours tend to show a deposition of calcium salts in the form of a fine powdering of basophilic granules as seen microscopically.

Tumours developed from the endometrium, comprising adenocarcinoma of the corpus uteri and adenocarcinoma of the portio endocervicalis, are composed of tall columnar cells, somewhat resembling those of the gastrointestinal tract, but showing ciliation. This serves as a point of differentiation.

Anaplastic forms of adenocarcinoma. examples where an alveolar, papilliform or? cribriform morphology is not apparent, histological differentiation may be more difficult. Here again some assistance may be obtained by the application of the mucicarmine staining reaction. The presence in a metastatic deposit of isolated cells of ' signet ring' type is strongly indicative of gastrointestinal carcinoma. Such cells are usually strongly positive to mucicarmine. Attenuated deposits of this type, in which the lymph node is diffusely but sparsely infiltrated with a few isolated signet ring cells are encountered particularly in linitis plastica. A similar histological picture is seen in the Krukenberg ovary.

Another form of adenocarcinoma is developed when the malignant epithelium reverts from its more usual functional regression and manifests functional hyperactivity with the production of large quantities of mucinoid exudate. These 'colloid' or 'mucoid' deposits are readily recognized in a lymph 
node. The malignant epithelial cells may be present in the form of well defined alveoli or as isolated units which have become distorted and compressed by the exuberance of their own secretory activity. The finding of such a deposit calls for a coordination of the clinical findings and the anatomical site of the node involved as adjuvant agents in determining the site of the primary neoplasm. Should oxyntic cells happen to be present the histological diagnosis of gastric carcinoma is established. It is necessary to bear in mind that the morphology of a lymph node metastasis serves merely as a guide to the anatomical site of the primary neoplasm and that the cytology of the primary growth and of its metastatic deposits are often by no means facsimiles.

If a metastatic deposit of anaplastic carcinoma is cytologically recognized as an adenocarcinoma, a weakly positive mucicarmine reaction suggests a malignant ovarian cystadenoma.

Adenocarcinoma of the gall bladder and biliary passages of the pancreas and of the urachus are composed of low cubical epithelium and are readily distinguishable from tumours of the gastrointestinal tract. The differentiation of individual members of this group is based on the presence of cilia in adenocarcinoma of the biliary passages and on the presence of mucin secretion in adenocarcinoma of the urachus. Anatomical considerations also assist in differential diagnosis.

The sialomata or tumours of the salivary glands form an important group which require careful differentiation. Though the majority are of slow development they must be regarded as essentially malignant tumours some of which produce lymph node metastases.

Sialomata, formerly known as ' mixed parotid tumours' or 'myxo-chondro-endotheliomata' are now recognized as being adenomata or adenocarcinomata of the epithelium of the ducts of the salivary glands. Their heterogeneous histological appearance is explained by the presence of a matrix of mucous tissue containing characteristic stellate cells which give an appearance of pseudocartilage. Nevertheless the members of this group are sufficiently typical in appearance as to enable their metastatic deposits to be appreciated without difficulty. Four main varieties are distinguishable - the alveolar, the pseudocartilaginous, the para buccal and the basal celled, but there may be admixtures of any combination of varieties. In the alveolar type there are well defined alveoli lined by low cubical or attenuated epithelium. In the pseudocartilaginous type the epithelial cells proper to the tumour undergo modification and compression as a result of the products of their own secretory hyperactivity. In the para buccal type very characteristic vesicles containing reticulated mucinoid secretion predominate. in the basal celled type the appearance is strikingly similar to that of a basal celled carcinoma of the skin. The latter tumour, however, does not give rise to lymph node metastases.

\section{Melanoma}

Metastatic deposits of the more highly pigmented forms of the melanomata are readily recognizable microscopically on account of the ease with which melanin pigment is demonstrable by specific cyto-chemical tests. With some of the achromic forms, however, considerable difficulty is likely to be encountered unless particular attention is paid to certain characteristic and peculiar properties appertaining to this wide group of highly malignant tumours.

It must be appreciated that all malignant tumours in which melanin is demonstrable are not to be included in the specific group comprising the melanomata. Fortuitous pigmentation is not infrequently met with in squamous carcinoma, basal celled carcinoma, endothelioma and fibrosarcoma.

The true melanomata are malignant tumours derived from neuroepithelium in connection with nerve end organs in the dermis, in the uveal tract, in the meninges, in the nasal fossae, and occasionally in the epidermis. The wide diversity of histological structure of these tumours may be correlated with the diverse types of nerve end organ that are present in these anatomical sites.

In metastatic tumour deposits in lymph nodes' which are pigmented, the nature of the pigment must be investigated by a series of cyto chemical reactions. It is a safe procedure 
to have several sections of the excised node prepared and stained by special technical methods, as follows :-

(a) Haematoxylin and eosin.

(b) Preliminary bleaching with oxalic acid and potassium permanganate, prior to various staining methods.

(c) Impregnation with ammoniacal silver solution to accentuate melanin. A suitable method of counter staining is the application of metanil yellow and neutral red.

(d) Application of the Prussian blue and Turnbull's blue reactions for iron containing pigments.

(e) Combined silver impregnation and staining for iron containing pigments.

The correct interpretation of the staining reactions in sections treated by the above methods requires care and experience. It must be borne in mind that a yellow, brown or black pigment which is visible in routine haematoxylin and eosin preparations may prove to be melanin, blood pigment free from iron, iron containing blood pigment, carbon, or pigment mechanically introduced, as in tattooing or in argyria. It is to be emphasized that such specific pigments may be present separately or in various admixtures.

If the pigment visible in the routine preparations (a) is completely bleached by the treatment (b), it may be melanin or an artifact resulting from a precipitation in blood clot in formalin fixed preparations. Differentiation here depends on the dense blackening of melanin by silver impregnation. Blood pigments are stained blue by the appropriate reagents, while carbon pigment is inert.

The application of Bloch's 'Dopa' reaction is useful for demonstrating the presence of non-pigmented melanogen in achromic tumours. It depends upon the presence of an oxydase which is obtained from the embryo of the broad bean and which converts the colourless melanogen into the pigment melanin.

A point of importance in arriving at a diagnosis of malignant melanoma is the dis- tribution and appearance of melanin pigment in melanoblasts and chromatophores respectively. In melanoblasts-that is in the cells of the malignant parenchyma - the pigment is present in the cytoplasm in the form of delicate rods or coccus-like bodies of yellowish or golden brown colour. In the chromatophores-that is in the wandering histiocytes of the part-the melanin is present in large coarse confluent globules of dark brown or black appearance. Consequently the more highly pigmented parts of a melanoma owe their blackness to the presence of chromatophores rather than melanoblasts. It is important to realize that if a lymph node when removed for biopsy examination appears dark in colour, it does not necessarily imply the presence of a metastatic deposit of melanoma. The pigmentation may be due merely to the presence of large numbers of chromatophores which have migrated from the site of the primary tumour.

In achromic forms of melanoma, recognition of specific types is sometimes easy. These bear the general resemblance to nerve end organs and classical examples are shown in the photomicrographs. There is one type, however, which appears histologically as a compact and uniform field of round cells. The differential diagnosis lies between seminoma, reticulosarcoma, anaplastic carcinoma and pinealoma. In seminoma, there are characteristically zones of focal necrosis, haemorrhage and of lymphocytic infiltration. Reticulosarcoma shows an intercellular matrix on staining with connective tissue and reticulin stains. Anaplastic carcinoma shows no intercellular matrix but may have an interlobular stroma and the presence of intercellular protoplasmic bridges may be demonstrable under high power magnification. Pinealoma is characterized by its mozaic morphology

A diagnostic point of considerable utility is the fact that the majority of achromic melanomata do, in fact, contain a small amount of melanin pigment. This may be limited to occasional groups of parenchymal cells or to isolated histiocytes or chromatophores. Accentuation by the application of ammoniacal silver solution renders the presence of the pigment obvious. In many cases it is necessary to make use of the oil immersion objective. 


\section{Bronchial Carcinoma}

The most characteristic form is the 'oat celled carcinoma.' When metastatic deposits of this form occur in lymph nodes, they are readily recognizable. The component cells are found in compact masses and may at first sight present superficial resemblance to lymphocytes. Critical examination, however, reveals the presence of short conical processes which occasionally may assume a more elongated or spindle form. The application of reticulum staining fails, of course; to reveal any intercellular matrix. When this type of metastatic deposit is found on histological examination of a lymph node, the diagnosis of the primary source of origin is readily arrived at from the morphological appearance of the cells. There are, however, other forms of primary bronchial carcinoma which may assume a frankly keratinized squamous character or which may be adenocarcinomata. In the latter type, it may be possible to recognize the specific characteristics of ciliated respiratory epithelium.

\section{Differential Diagnosis of Round Cell}

\section{Tumours}

There are many forms of metastatic deposits and of primary malignant neoplasms occurring in lymph nodes which on cursory examination appear to be composed of round cells. The differentiation depends upon a proper appreciation of the detailed cytology of such tumours together with a consideration of the stroma and reticulum and of the presence of degenerative changes and evidence of secretory activity, pigment formation and so on. The following include those tumours, both primary and metastatic, which may give rise to confusion in histological interpretation :-

(a) Lymphosarcoma.

(b) Lymphoblastic lymphosarcoma.

(c) Reticulosarcoma.

(d) Seminoma.

(e) Achromic melanoma.

(f) Ewing's tumour of bone.

(g) Pinealoma.

(h) Plasmacytoma.

(i) Oat celled bronchial carcinoma.

(j) Certain metastic tumours of the gastrointestinal tract and certain metastatic tumours of the ovary.

The features of the foregoing group have been described and discussed in some detail in connection with the differential diagnosis of specific cases of lymph node enlargement.

\section{BOOKS RECEIVED}

The Editorial Board acknowledge with thanks the receipt of the following volumes. A selection from these zill be made for review.

' Gas and Air Analgesia.' By R. J. Minnitt, M.D., D.A., 3rd Ed. (Pp. viii +80 , 19 illustrations, 5s.) Barlliere, Tindall and Cox.

'Practical Textbook of Leprosy.' By R. G. Cochrane, M.D., F.R.C.P., D.T.M. and H. (Pp. 282, 42s.) Geoffrey Cumberlege. Oxford University Press.

'Diseases of the Nervous System.' By. W. Russell Brain, D.M., F.R.C.P. 3rd Ed. (Pp. 987, 37s. 6d.) Geoffrey Cumberlege. Oxford University Press.
'Dying, Apparent Death and Resuscitation.' By S. Jellinek, M.D. (Pp. viii $+26 \dot{3}$, ros. 6d.) Bailliere, Tindall and Cox.

Supplement to Lewis's Library Catalogue, 19441946. (Pp. iv $+176,5 \mathrm{~s}$. To subscribers to the Library, 2s. 6d.)

Air Ministry. Handbook of Preventive Medicine. Air Publication ap 1269B. (Pp. 213, 7s. 6d.) H.M. Stationery Office, London.

Monograph on the Progress of Research in Holland : "Modern Development of Chemotherapy.' By E. Havinga, H. W. Julius, H. Veldstra, K. C. Winkler. (Pp. 175, 15s.). Published by Elsevier. Distributors : Cleaver-Hume Press. 